\title{
Uptake of Genetic Testing and Pre-Test Levels of Mental Distress in Norwegian Families with Known BRCA1 Mutations
}

\author{
Jon G. Reichelt ${ }^{1,2, \#}$, Alv A. Dahl ${ }^{2}$, \\ Ketil Heimdal $^{1}$ and Pål Møller ${ }^{1}$ \\ ${ }^{1}$ Unit of Medical Genetics, The Norwegian \\ Radium Hospital, University of Oslo, 0310 \\ Oslo, Norway \\ ${ }^{2}$ Department of Psychiatry, Aker Hospital, \\ University of Oslo, 0320 Oslo, Norway
}

\begin{abstract}
: 232 family members from 27 Norwegian families with BRCAl mutations were offered genetic testing. 180/232 (78\%) chose to be tested, 14/232 (6\%) have not yet decided and 38/232 (16\%) declined. All 232 persons were invited to fill in the following questionnaires when offered testing: Impact of Event Scale (IES), Hospital Anxiety and Depression Scale (HADS), General Health Questionnaire (GHQ-28) and Beck Hopelessness Scale (BHS). 207/232 (89\%) responded to the questionnaires. Of those declining to be tested 23/38 $(61 \%)$ answered the questionnaires compared to $170 / 180(94 \%)$ of those wanting the test $(p<0.0001)$.

A higher proportion of females with a history of cancer than females without such a history had abnormal scores on the IES-intrusion and GHQ questionnaires $(\mathrm{p}<0.001)$. Healthy females who were deciding on predictive testing had the same or lower prevalence of mental distress compared to the general population, between $4.3 \%$ and $18.0 \%$ as measured by the different questionnaires. Males did not differ from healthy females on any of the measures.

According to their HADS scores, women without a history of cancer deciding on predictive testing for breast-ovarian cancer had lower or equal levels of mental distress compared to the general population. The high uptake of genetic testing combined with the
\end{abstract}

\footnotetext{
\# Corresponding author: Dr. Jon G. Reichelt, MD, Section of Medical Genetics, The Norwegian Radium Hospital, Montebello, 0310 Oslo, Norway, Tel.: +47 22934000; Fax: +47 22935219; E-mail: jon.reichelt@klinmed.uio.no
}

lower than normal prevalence of mental distress indicates that the activity may continue as practised, awaiting longitudinal data concerning the levels of mental distress after genetic testing.

\section{INTRODUCTION}

The finding of founder mutations of BRCA1 in Norwegian families with heritable breast and ovarian cancer has made it possible to identify a number of mutation carriers, and offer genetic testing to their relatives. Previously these relatives were given genetic counselling, on the basis of clinical criteria, that gives female members in the same kindred an increased risk of contracting cancer. Genetic testing provides more certain knowledge, with both the possibility of good news (negative test) - risk comparable to that of the rest of the population - and the possibility of bad news (positive test) that increases the likelihood of contracting cancer. The advent of genetic testing makes it of interest to know more about recruitment to testing and how family members cope with this opportunity.

Previous reports have shown that interest in testing has been high but that a limited number of relatives actually go on to be tested [11], and a high number of family members report that they anticipate negative psychological reactions to a positive test [14]. In contrast to the public debate on assumed adverse effects, no significant psychological disturbances among the tested family members have actually been reported. A summary of the literature has been given [2]. Our knowledge of predictive genetic testing for adultonset diseases is based largely on experience with incurable diseases, like Huntington's disease. A 
review concludes that both carriers and noncarriers experience short-term emotional reactions, but no long-term reactions have been revealed [13]. Whether these findings are relevant for a different disease with lower penetrance and a possibility of cure, such as inherited breast cancer, has not been examined. As the first report in a longitudinal study concerning the psychological effects of genetic testing, we here report on the uptake of testing in Norwegian families with demonstrated mutations in BRCA1 who have been offered testing, their compliance with psychosocial questionnaires, their prevalence of mental distress and levels of anxiety and depression at the time when they are offered a test.

\section{MATERIAL AND METHODS}

The population studied is drawn from our cancer clinic cases, including more than 1000 breast cancer kindreds as clinically defined. Most of the families are self-referred, and we have demonstrated the underlying BRCA1 mutation in a number of families. We have earlier published our findings of founder mutations and the results of our program for early detection of breast and ovarian cancer $[3,4,12]$. As of December 31st 1998, 232 members of 27 families with demonstrated BRCA1 mutations had been offered genetic testing. $156(67 \%)$ were women who had not had cancer, $46(20 \%)$ were men (none had experienced cancer) and 30 (13\%) were women who had had breast and/or ovarian cancer. As agreed at earlier genetic counselling sessions, they were offered genetic testing when it became available. At the same time as they were offered testing they received written information about the different aspects of testing; they were invited to pre-test counselling, and they were also invited to complete the psychosocial questionnaire described below before coming to counselling. One written reminder was sent to those not responding to the first request after 8 weeks.

Levels of anxiety and depression were measured with the Hospital Anxiety and
Depression Scale (HADS) [7]. The questionnaire has 14 items, half of which reflect anxiety and half depression. HADS gives overall scores for anxiety $(\min 0, \max 21)$ and depression $(\min 0$, max 21). A score of 8 or higher on each sub-scale indicates possible "caseness", and a score of 11 or higher definite "caseness". We applied the lower threshold, to increase the sensitivity for possible "cases".

The General Health Questionnaire (GHQ-28) is focused on psychosocial distress and subjective wellbeing. We used the "scaled version" which has 28 items divided into four sub-scales, social functioning, somatic symptoms, anxiety/sleep disturbances and depression. To identify cases we applied "simple" scoring, in which each item is given a score based on the symptom both being present and being present to a greater extent than usual $(0,0,1,1)$. The threshold is usually between 5 and 6 , but it has been suggested to use a threshold between 7 and 8 when screening a population with a somatic disease [6,9]. We applied both in this study, since one subgroup had a history of cancer and the other two had not.

Degree of hopelessness was measured with the Beck Hopelessness Scale (BHS), a questionnaire that has 20 items. BHS scores can vary from 0 to 20 , with scores of 9-13 indicating moderate hopelessness and scores $\geq 14$ indicating severe hopelessness [1]. We applied the lower threshold of between 8 and 9 for "caseness", in order to identify all possible "cases" with high sensitivity.

The Impact of Event Scale (IES) is a questionnaire that measures psychological distress on two sub-scales, "intrusion" that refers to intrusively experienced images, thoughts, feelings and dreams and "avoidance" that refers to consciously recognised avoidance of certain feelings, ideas or situations. The intrusion sub-scale has 7 items, the avoidance sub-scale has 8 items, and each item has a score of 0-5. A score on each sub-scale of 9-19 denotes a moderate level of distress and a score of $\geq 20$ denotes more severe distress [8]. We applied the threshold of a score of $>20$, because low to moderate stress levels were expected to be frequent in individuals offered genetic testing for an inherited disease.

All returned questionnaires with less than $90 \%$ 
of each subscale properly answered, were excluded from analysis of that subscale. When less than $10 \%$ of the items were missing, missing items were given a score according to the mean of the answered items.

\section{RESULTS}

As of May 1st 1999, 180 out of 232 family members have received the results of genetic testing (78\%), 14/232 (6\%) have not decided yet and 38/232 (16\%) have declined. Of 158 females without a history of breast and/or ovarian cancer 120 had opted for testing (76\%).

Total compliance in answering the questionnaires was 207/232 (89\%). Compliance among females without a history of cancer was $142 / 158(90 \%)$. A higher fraction of those wanting the test answered the questionnaires, $170 / 180(94 \%)$, than of those not wanting the test, $23 / 38(61 \%)(\mathrm{p}<0.0001)$. This was also true for females without a history of cancer. $114 / 120(95 \%)$ of those who wanted testing answered the questionnaires compared to 19/29 $(66 \%)$ of those who did not want testing $(\mathrm{p}<0.0001)$

Details of distribution of scoring on each questionnaire are given in Table 1. For females without a history of cancer we found that clinical levels of mental distress varied from 6/142 $(4.3 \%)$ on the HADS-Depression sub-scale to $26 / 142(18.0 \%)$ on the HADS-Anxiety sub-scale.
All results from the other questionnaires were within this range. For females with a history of cancer the proportion with mental distress varied from $3 / 25(12.5 \%)$ on the HADS-Depression to $10 / 25(41.7 \%)$ on the IES-Intrusion (IES-I) subscale.

There was a statistically significant $(\mathrm{p}<0.01)$ higher number of cases among the females with a history of cancer compared with females without such a history, as measured by the IES-I and the GHQ-28. The number of cases was 10/25 $(41.7 \%)$ versus $15 / 142(10.7 \%)$ on the IES-I and $9 / 25(37.5 \%)$ versus $18 / 142(12.7 \%)$ or $11 / 142$ (7.8\%) on the GHQ, depending on the threshold value. There were no significant differences between males and females without a history of cancer on any of the questionnaires.

\section{DISCUSSION}

The uptake of genetic testing was as high as $78 \%$ in our study: Previous reports give uptake rates between $22 \%$ [5] and 43\% [11]. A high uptake rate can be understood as a high degree of trust that inherited breast cancer may be cured, in confidentiality concerning the test results, and in the fact that Norway has a public health care system basically without cost for the patients. They are offered life saving health services for free, and their access to insurance/bank loans has not so far been affected. The high compliance rate in terms of return of completed

Table 1

Distribution of results obtained from the 207 (89\%) out of 232 who returned the questionnaires stratified on females without cancer, females with cancer and males. Mean and SD for distribution are given, as well as number of cases with abnormal high scores (see text for caseness estimation)

\begin{tabular}{|c|c|c|c|c|c|c|c|c|c|c|}
\hline \multirow[b]{2}{*}{ Scale } & \multirow[b]{2}{*}{ Cut-off } & \multicolumn{3}{|c|}{ Females without cancer $(n=142)$} & \multicolumn{3}{|c|}{ Females with cancer $(n=25)$} & \multicolumn{3}{|c|}{ Males $(n=40)$} \\
\hline & & Mean (SD) & $\begin{array}{c}\text { "cases" } \\
\mathrm{n}(\%)\end{array}$ & e.c. $^{2}$ & Mean (SD) & $\begin{array}{c}\text { "cases" } \\
\text { n }(\%)\end{array}$ & e.c. ${ }^{2}$ & Mean (SD) & $\begin{array}{c}\text { "cases" } \\
\text { n (\%) }\end{array}$ & e.c. ${ }^{2}$ \\
\hline IES-I & $>=20$ & $8.7(7.2)$ & $15(10.7)$ & 2 & $17.4(8.6)$ & $10(41.7)^{1}$ & 1 & $6.8(8.1)$ & $3(7.9)$ & 2 \\
\hline IES-A & $>=20$ & $8.2(7.2)$ & $14(10.1)$ & 3 & $11.9(8.4)$ & $5(20.8)$ & 1 & $8.1(7.7)$ & $3(7.9)$ & 2 \\
\hline HADS-A & $>=8$ & $4.5(3.8)$ & $26(18.0)$ & 1 & $5.8(3.6)$ & $7(29.2)$ & 1 & $3.7(3.5)$ & $6(15.4)$ & 1 \\
\hline HADS-D & $>=8$ & $1.8(2.2)$ & $6(4.3)$ & 1 & $3.0(2.8)$ & $3(12.5)$ & 1 & $2.4(2.9)$ & $4(10.3)$ & 1 \\
\hline GHQ-28 & $>=6$ & $2.3(3.8)$ & $18(12.7)$ & 0 & $4.7(5.2)$ & $9(37.5)^{1}$ & 1 & $1.9(4.6)$ & $5(12.8)$ & 1 \\
\hline GHQ-28 & $>=8$ & $2.3(3.8)$ & $11(7.8)$ & 0 & $4.7(5.2)$ & $9(37.5)^{1}$ & 1 & $1.9(4.6)$ & $4(10.3)$ & 1 \\
\hline BHS & $>=9$ & $4.0(2.6)$ & $7(5.1)$ & 5 & $5.6(3.5)$ & $4(16.7)$ & 1 & $4.4(2.5)$ & $4(10.0)$ & 0 \\
\hline
\end{tabular}

${ }^{1} \mathrm{p}<0.01$ compared with females without cancer

2 e.c.: excluded cases due to incomplete answers 
questionnaires in our study (89\%) give us results that are more representative, compared to the relatively low compliance in other studies [2]. Our sample size makes variations due to small numbers unlikely. The results obtained are, therefore, considered representative for the population studied.

In a population study of 33,168 females and 29,328 males aged 20-89 years in Norway [Stordal et al. Submitted] HADS-Anxiety $(\geq 8)$ was $17.3 \%$ among females and $12.1 \%$ among males. Mean score in females was 4.47 (SD 3.45) and mean score in males was 3.87 (SD 3.08). The prevalence of depression in the same study was $10.4 \%$ among females on HADS-Depression $(\geq 8)$ and $10.8 \%$ among males. Mean score in females was 3.33 (SD 3.04) and mean score in males was 3.54 (SD 3.03). Comparing these results with the present study, we found a lower level of mental distress measured by HADS-Depression $(\mathrm{x} 2=5.2, \mathrm{p}<0.025)$, and no difference as measured by HADS-Anxiety $(\mathrm{x} 2=0.1, \mathrm{p}>0.1)$ among women without a history of cancer.

The high levels of "caseness" on GHQ-28 among females with a history of cancer could be caused by somatic symptoms, and by the fact that they are experiencing distress both from the history of cancer, the possibility of having increased risk of another cancer and the possibility of having given their children the mutation. The interpretation of mental distress caused by the cancer itself is in keeping with a previous report on breast cancer patients [15]. In a study of post-traumatic distress in primary breast cancer in Norway the prevalence of "caseness" on GHQ-28 with a threshold of $7 / 8$ was $24 \%$ six weeks post surgery and $14 \%$ one year post-surgery, with a mean score one year post-surgery of 3.2 (SD 5.6). Females without a history of cancer again showed low levels of "caseness" in our study.

A study of adaptation to presymptomatic testing for late onset disorders [4] found a pretest mean score on BHS in individuals $(\mathrm{n}=23)$ tested for familial adenomatous polyposis (FAP) of 4.9 (SD 3.1) and in individuals tested for hereditary breast and ovarian cancer (HBOC) $(n=10)$ of 3.9 (SD 1.4). Our findings do not differ from these results.

The IES is considered to be the most sensitive of measures used in the study of effects of stressful events, and under stressful circumstances such a sensitive instrument should indicate low and moderate stress symptoms. For this reason we used a high threshold for defining "caseness" with this instrument. As expected, we found moderately raised levels of distress measured on IES in the females without a history of cancer at the time of deciding on genetic testing. Females with a history of cancer scored significantly higher on IES-I, suggesting an additive effect of the history of cancer. More detailed analysis of our findings must await follow-up data and longitudinal evaluation. The results so far are in keeping with previous reports. Longitudinal studies of healthy persons' responses to a traumatic event demonstrate higher mean intrusion compared to avoidance symptoms [10], as findings for females with a history of cancer show in this study. In the study of posttraumatic distress in primary breast cancer in Norway Tjemsland et al. [15] found medium scores on IES (9-19) in 37\% (IES-I) and 46\% (IES-A) and high scores $(\geq 20)$ in $44 \%$ and $29 \%$ respectively at the time after diagnosis but before surgery. The results one year after surgery were medium scores (9-19) in 37\% (IES-I) and 29\% (IES-A), and high scores $(\geq 20)$ in $9 \%$ and $10 \%$ respectively.

In summary, our findings show an apparent lack of adverse psychological reactions to our offer of predictive genetic testing in both males and females without a history of cancer, but raised levels of mental distress and number of cases among females with a history of cancer. We also found an uptake of testing much higher than in any previous report and this may be an effect of self-referral to our health-service. Whatever the explanation of these results, they are as hoped for, when considering the future of offering predictive genetic testing for curable forms of cancer to healthy members in families with demonstrated mutations. The questionnaires used have previously been validated both 
nationally and internationally, and the number of responders was large enough to escape uncertainty relating to variation in small numbers. We conclude that the high uptake of genetic testing and the lack of adverse mental distress found on pre-test questionnaires, answered with high compliance by healthy family members indicate that the activity of predictive testing may continue without undue fear of adverse psychological effects in those offered testing. Continuous follow-up of the patients described will give results on both short and long term effects of our intervention both for those who choose to be tested and for those who choose not to undergo predictive testing.

\section{References}

[1] Beck, A.T., Weismann, A., Lester, D. and Trexler, L. The measurement of pessimism: the hopelessness scale. J. Cons. Clin. Psychol. 42, (1974) 861-865.

[2] Dudok deWit, A.C., Tibben, A., Duivenvoorden, H.J., Niermeijer, M.F., Passchier, J. et al. Predicting adaptation to presymptomatic DNA testing for late onset disorders: who will experience distress? J. Med. Genet. 35, (1998) 745-754.

[3] Dørum, A., Kristensen, G.B., Abeler, V.M., Trope, C.G. and Møller, P. Early detection of familial ovarian cancer. Eur. J. of Cancer 10, (1996) 1645-1651.

[4] Dørum, A., Møller, P., Kamsteeg, E.J., Scheffer, H. et al. A BRCA1 founder mutation, identified with haplotype analysis, allowing genotype/ phenotype determination and predictive testing. Eur. J. of Cancer 14, (1997) 2390-2392.

[5] Goelen, G., Teugels, E., Bonduelle, M., Neyns, B. and De Greve, J. High frequency of BRCA1/2 germline mutations in 42 Belgian families with a small number of symptomatic subjects. J. Med. Genet. 36, (1999) 304-308.
[6] Goldberg, D.P. and Williams, P. A user's guide to the General Health Questionnaire. NFR Nelson, Windsor, 1988.

[7] Hermann, C. International experiences with the anxiety and depression scale - a review of validation data and clinical results. $J$. of Psychosom. Res. 42, (1997) 17-41.

[8] Horowitz, M.J., Winer, N. and Alvarez, W. Impact of Event Scale: a measure of subjective stress. Psycosom. Med. 41, (1979) 209-218.

[9] Ibbotson, T., Maguire, P., Selby, P., Priestman, T., Wallace, L. Screening for anxiety and depression in cancer patients: The effect of disease and treatment. Eur. J. Cancer 30A, (1994) 37-40.

[10] Karlehagen, S., Malt, U.F., Hoff, H., Tibell, E., Herrstromer, U., Hildingson, K. and Leyman, H. The effect of major railway on the psychological health of train drivers-II. A longitudinal study of the one-year outcome after the accident. $J$. Psychosom. Res. 37, (1993) 807-817.

[11] Lerman, C., Narod, S., Schulman, K. et al., BRCA1 testing in families with hereditary breastovarian cancer. JAMA 275, (1996) 1885-1892.

[12] Møller, P., Mæhle, L., Heimdal, K., Dørum, A. et al. Prospective findings in breast cancer kindreds: annual incidence rates according to age, stage at diagnosis, mean sojourn time, and incidence rates for contralateral cancer. The Breast 7, (1998) 5559.

[13] van 't Spijker, A. and ten Kroode, H.F.J. Psychological aspects of genetic counselling: A review of the experience with Huntington's disease. Patient Education and Counselling 32, (1997) 33-40.

[14] Struewing, J.P., Lerman, C., Kase, R.G., Giambarresi, T.H. and Tucker, M.A. Anticipated uptake and impact of genetic testing in hereditary breast and ovarian cancer families. Cancer Epidemiology, Biomarkers \& Prevention 4, (1995) 169-173.

[15] Tjemsland, L., Søreide, J.A. and Malt, U.F. Posttraumatic distress symptoms in operable breast cancer III: Status one year after surgery. Breast Cancer Research and Treatment 47, (1998) 141-151. 


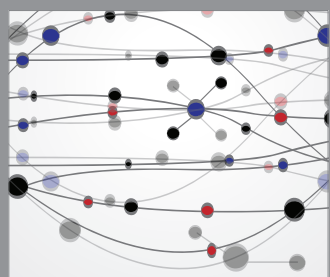

The Scientific World Journal
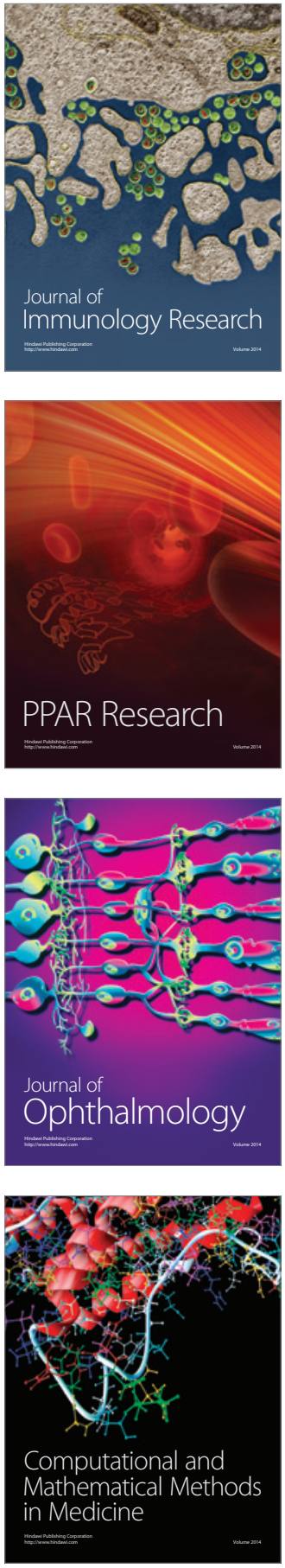

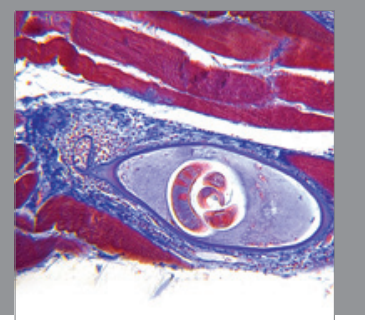

Gastroenterology

Research and Practice
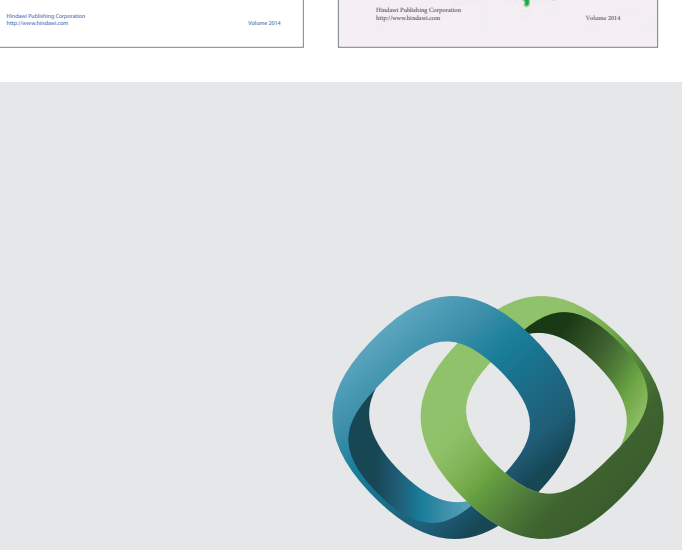

\section{Hindawi}

Submit your manuscripts at

http://www.hindawi.com
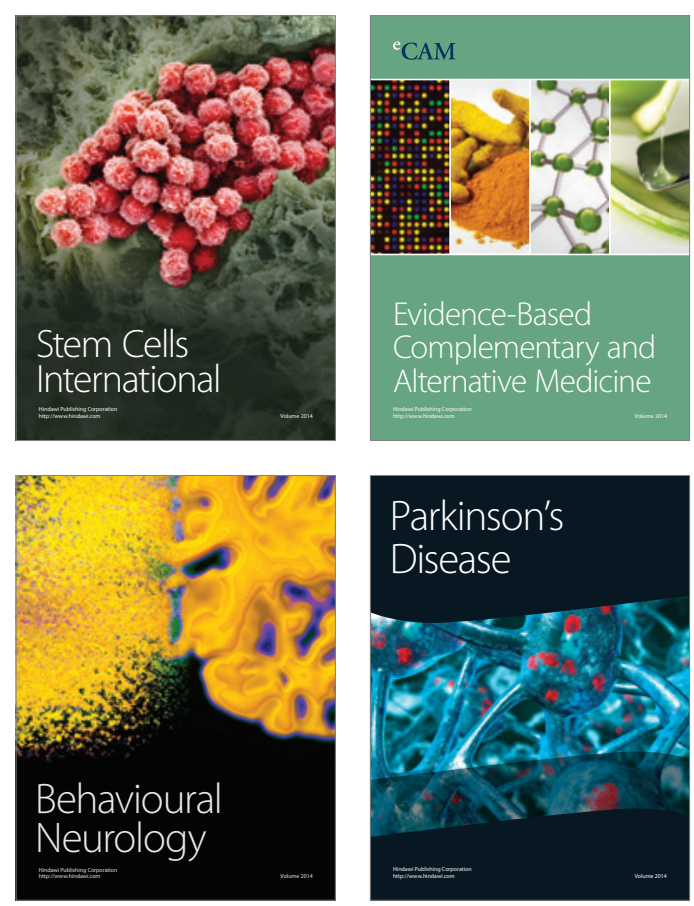

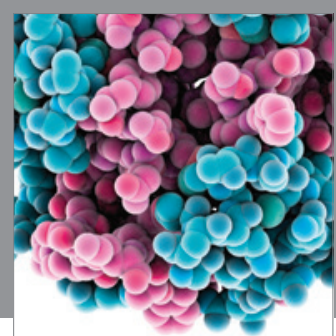

Journal of
Diabetes Research

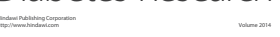

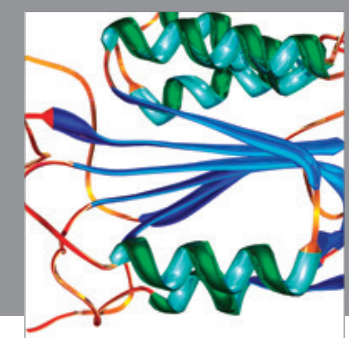

Disease Markers
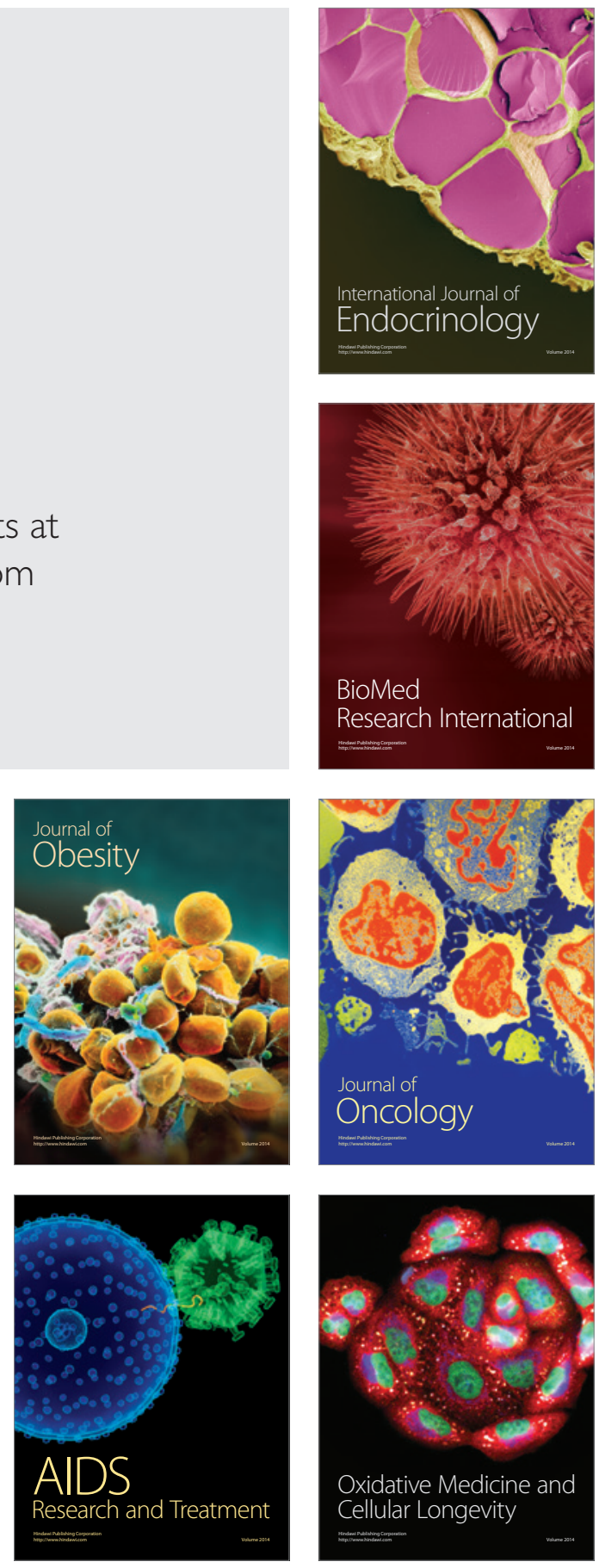